\title{
¿Es actualmente el Score de Apgar un predictor útil de muerte neonatal?
}

The Continuing Value of the Apgar Score for the assessment of newborn infants. Casey B, Mclntire D, Leveno K. N Eng J Med 2001 Feb $7: 344: 467-471$

\section{Objetivo}

Examinar si el score de Apgar es un predictor de muerte en el período neonatal y comparar su utilidad como predictor en relación al valor de $\mathrm{pH}$ en arteria umbilical

Diseño

Estudio de cohorte retrospectivo

Lugar

Base de datos de Parkland Hospital, Dallas (EE UU), Departamento de Obstetricia y Pediatría

\section{Pacientes}

El estudio incluyó 151891 recién nacidos vivos (RN), con edad gestacional igual o mayor a 26 semanas. Se excluyeron gemelos o pacientes con malformaciones mayores, identificadas en sala de partos o mediante autopsia.

\section{Evaluación de factores pronóstico}

Se evaluaron los nacimientos ocurridos entre 1988 y 1998 . Se consideró la puntuación del score de Apgar al 1er y 5 to minuto, asignada rutinariamente por el neonatólogo o el enfermero encargado de la recepción en la sala de partos, de acuerdo a que se tratase o no de un nacimiento de alto riesgo. Los datos de gases sanguíneos y $\mathrm{pH}$ de las muestras de la arteria umbilical fueron obtenidos en 145627 niños.

Medición del resultado principal

La evaluación principal fue la muerte ocurrida en los primeros 28 días de vida, la cual se relacionó con la puntuación de Apgar y el $\mathrm{pH}$ al nacer, considerando en el análisis la población de prematuros ( $<$ de 36 semanas) y la de nacidos a término.

\section{Resultados principales}

En la tabla se presentan la incidencia y el riesgo relativo de muerte neonatal de acuerdo a la puntuación de Apgar al 5to minuto en ambas poblaciones (prematuros y término). El estudio no muestra los datos correspondientes al 1er minuto por considerarlos de escaso valor.

\begin{tabular}{|l|l|l|l}
\hline Apgar al 5to minuto & $\begin{array}{l}\text { No RN } \\
\text { (Total 151891) }\end{array}$ & Muertes o/00 & $\begin{array}{l}\text { Riesgo Relativo } \\
\text { (CG95\%) }\end{array}$ \\
\hline $0-3$ & Pret 92 & 315 & $59(40-87)$ \\
\hline $4-6$ & Term 86 & 244 & $1460(835-2555)$ \\
\hline & Pret 556 & 72 & $13(9-20)$ \\
\hline $7-10$ & Term 561 & 9 & $53(20-140)$ \\
\hline & Pret 12751 & 5 & 1 \\
\hline & Term 131581 & 0,2 & 1 \\
\hline
\end{tabular}

Para RN de término y pretérmino el riesgo de morir es significativamente mayor a menor puntación de Apgar. El Apgar de 0 - 3 fue comparativamente mejor predictor de muerte que la acidemia $(\mathrm{pH}<7)$. Si ambos índices (Apgar + acidemia) se combinan el riesgo se incrementa: RR (IC95\%)= $3204(1864-5508)$ en RN de término y $102(65-160)$ en pretérmino.

\section{Conclusiones}

E| Apgar continúa siendo un score válido para predecir muerte neonatal.

\section{Comentario}

Virginia Apgar diseñó en 1952 este sistema de puntuación con el objetivo de valorar el estado clínico del RN luego de nacer. Este score simple y rápido surge de la suma de cinco componentes: frecuencia cardíaca, esfuerzo respiratorio, tono muscular, irritabilidad refleja y color, cada uno de los cuales recibe entre 0 a 2 puntos, al 1er y 5 to minuto de vida. Un Apgar mayor de 7 indica que la condición neonatal es muy buena. La Dra Apgar asigna a este sistema el valor de comparar prácticas obstétricas y de estimar la probabilidad de sobrevida neonatal; posteriormente, lo propone como indicador de la reanimación neonatal. 1 -2

Desde la creación del Apgar hace 50 años, la neonatología ha sufrido cambios profundos tanto en la evaluación diagnóstica como en las posibilidades de tratamiento de los recién nacidos. Surge de esto la pregunta: el Apgar hoy, es una medición útil u obsoleta? El estudio del Dr Casey y col. define el valor del Apgar en el contexto actual de la neonatología. Sus hallazgos son idénticos a los publicados hace 40 años por el Estudio Colaborativo de Parálisis Cerebral3: una fuerte asociación entre Apgar $\leq$ de 3, a los 5 minutos y muerte neonatal. Sin embargo, entre ambas publicaciones hay una significativa diferencia: los cambios en la práctica neonatal.
Mientras que en el estudio colaborativo la condición al 5 to minuto reflejaba probablemente la evolución natural, en este estudio de Casey un Apgar $\leq$ de 3 es indicativo de un niño severamente comprometido que no responde a los esfuerzos de resucitación del equipo neonatal. A pesar que el valor medio del score al 5 to minuto es menor a menor edad gestacional, la ocurrencia de muerte neonatal se relacionó significativamente con el Apgar, independientemente de la edad gestacional.

El Apgar bajo en RN de término mostró un riesgo de muerte ocho veces mayor en comparación con la acidemia grave.

En la actualidad existe consenso en que el Apgar no puede ni debe diagnosticar asfixia perinatal, ni indicar reanimación, ni pronosticar evolución neurológica ${ }^{4}$. Su uso en este marco para el cual no fue diseñado puede ser riesgoso.

Finalmente, como la mayoría de las herramientas diagnósticas a nuestro alcance, el Apgar es útil si su uso es adecuado y se conocen sus limitaciones. Después de 50 años, Virginia Apgar tenía razón: la observación clínica de los recién nacidos fue y sigue siendo extremadamente valiosa.

\section{Dra. Susana Rodríguez [ Unidad de Cuidado Intensivo Neonatal Hospital J P Garrahan ]}

\author{
Referencias
1 Apgar V. Proposal for new method of evaluation of newborn infant. Anesth Analg 1953; 32:260-7 \\ 1 Apgar V. Proposal for new method of evaluation of newborn infant. Anesth Analg 1953; 32:260-7
2 ApgarV, HoladayA, et al. Evaluation of the newborn infant - second report. JAMA 1958; 168: 1985-88 \\ 2 ApgarV, HoladayA, et al. Evaluation of the newborn infant - second report. JAMA 1958; 168: 1985-88
3 Drage JS et al. The apgar score as an index of neonatal mortality: a report from the Collaborative Study of cerebral palsy. Obstet Gynecol 1964; $24: 222-30$ \\ 3 Drage JS et al. The apgar score as an index of neonatal mortality: a report from the Collaborative Study of cerebral palsy. Obstet Gynecol
4 American Academy of Pediatrics, Committee on Fetus and Newborn. Use and Abuse of the Apgar Score. Pediatrics 1986; 78(6): 1148-9
}

